ORIGINAL ARTICLE

\title{
Multiple high risk HPV infections are common in cervical neoplasia and young women in a cervical screening population
}

\author{
K S Cuschieri, H A Cubie, M W Whitley, A L Seagar, M J Arends, C Moore, G Gilkisson, \\ E McGoogan
}

See end of article for authors' affiliations

Correspondence to:

Correspondence to: Virology Centre, Royal Infirmary of Edinburgh, 51 Little France Crescent, Edinburgh EHI 6 4SA', UK; kate.cuschieri@luht. scot.nhs.uk

Accepted for publication 30 June 2003

\begin{abstract}
Aims: If human papillomavirus (HPV) testing is to be included within cervical screening programmes, the importance of multiple HPV infections in cervical neoplasia needs to be determined. This study investigated the diversity of multiple HPV types in a routine cervical screening population, and assessed associations with cervical neoplasia.

Methods: Overall HPV prevalence, type specific prevalence, and extent of multiple infection were assessed in residual material from 3444 liquid based cytology samples, using real time GP5+/GP6+ polymerase chain reaction for screening and linear array assay for genotyping. HPV status was studied in relation to age and concurrent cytological evidence of dyskaryosis.

Results: Twenty per cent of samples were HPV positive. HPV type diversity was broad, and multiple HPV infections occurred in half of the HPV positive samples. Younger women were significantly more likely to harbour multiple high risk HPV (HR-HPV) infections. Infections with multiple HR-HPV types were found in $3.4 \%$ of samples negative for neoplasia and in $33.3 \%, 41.8 \%$, and $40.4 \%$ of samples with borderline, mild, or high grade dyskaryosis, respectively. Single HR-HPV infections were found in $4.9 \%, 38.6 \%$, $45.0 \%$, and $51.1 \%$ of negative, borderline, mild, or high grade dyskaryosis samples, respectively.

Conclusions: Multiple HR-HPV infections were most prevalent in young women. Multiple HR-HPV infections were not more frequent in high grade than in low grade cervical neoplasia, reflecting common sexual transmission of multiple HR-HPV. Prospective cohort studies linking sequential loss or gain of HPV types with cytological analysis are required to assess the impact of multiple HR-HPV infections on neoplastic progression.
\end{abstract}

T he implementation of cytology based cervical screening programmes has reduced the incidence of cervical carcinoma world wide. Consequently, the development of improved cytological preparation methods such as liquid based cytology (LBC) and thin layer slide preparation has been welcomed. The addition of high risk human papillomavirus (HR-HPV) testing may further enhance the accuracy of screening programmes. Accumulating evidence suggests that detection of persistent infection with the same HR-HPV type would highlight individuals at greater risk of disease progression, ${ }^{12}$ yet the extent and importance of multiple HR-HPV infections in the progression of cervical neoplasia and its management remain unknown. To investigate these issues it is necessary to use genotyping methods that can track the persistence of specific HPV types and detect infections with multiple HR-HPV types.

\begin{abstract}
"Accumulating evidence suggests that detection of persistent infection with the same high risk human papillomavirus (HR-HPV) type would highlight individuals at greater risk of disease progression, yet the extent and importance of multiple HR-HPV infections in the progression of cervical neoplasia and its management remain unknown"
\end{abstract}

A recent directive issued by the Scottish Executive has promoted the roll out of cervical LBC for use throughout primary care in Scotland. The residual material from LBC specimens is an ideal template for HPV testing. We have investigated the prevalence and diversity of HPV infection in a routine cervical screening population in Scotland using residual material from LBC specimens. The extent of multiple HPV infections was assessed and compared with cytological assessment of neoplasia. The results constitute the baseline data of a longitudinal study designed to investigate the impact of HPV detection, multiple HPV infection, and HPV type specific persistence on progression of cervical neoplasia.

\section{MATERIALS AND METHODS}

Sample collection and subjects

LBC has been in place since 1999 for 15 primary care practices in Edinburgh and, to date, over 23000 specimens have been received. The technique differs from the traditional Pap smear in that after the cervical sample is taken, rather than being deposited on a glass slide, it is rinsed into a vial containing an alcohol based cytological preservative solution. A small volume of the resulting cellular suspension is then processed by a robotic device to produce a flat layer slide. The technique has been claimed to enhance the accuracy of cytological assessment. It also has the advantage that the residual suspension, not used for cytology, can act as a template for microbiological testing. Local research ethics committee approval was granted for microbiological testing and informed consent was obtained. In 2000, we chose a subset of 3444 randomly selected specimens (age range, 16.578 years; mean age, 36.6) to determine HPV prevalence.

\footnotetext{
Abbreviations: HIV, human immunodeficiency virus; HPV, human papillomavirus; HR, high risk; LBC, liquid based cytology; LA, linear array; LR, low risk; PCR, polymerase chain reaction
} 
Primary care personnel performed specimen collection, flat layer slides were created by the ThinPrep ${ }^{\circledR}$ procedure, and cytological grading was performed according to British Society for Clinical Cytology guidelines. After cytology, residual cells in the specimens were centrifuged at $2800 \times \mathrm{g}$ for 10 minutes and stored as cellular pellets at $-70^{\circ} \mathrm{C}$ in a lysis buffer (Qiagen Ltd, Crawley, West Sussex, UK) before nucleic acid extraction and HPV detection.

\section{HPV detection and genotyping}

Briefly, automated nucleic acid extraction of cellular pellets was performed on a BioRobot $9604{ }^{\circledR}$ (Qiagen Ltd) using the 96 well silica column plates and reagents supplied within the QIAamp ${ }^{\circledR} 96$ DNA Swab BioRobot kit. The robotic protocol, which is optimised for LBC samples, was carried out as described previously. ${ }^{3}$ HPV detection was performed by real time polymerase chain reaction (PCR) using GP5+/GP6+ consensus PCR primers with the LightCycler ${ }^{\mathrm{TM}}$ apparatus, as described previously. ${ }^{4}$ Genotyping of HPV positive samples was performed via linear array (LA) hybridisation assay, ${ }^{5}$ which involved the hybridisation of PCR products to a strip containing two levels of $\beta$ globin control probes, $18 \mathrm{HR}-\mathrm{HPV}$ probes $(16,18,26,31,33,35,39,45,51,52,55,56,58,59,68$, $73,82,83)$, and nine low risk HPV (LR-HPV) probes $(6,11$, 40, 42, 53, 54, 57, 66, 84) (E McGoogan, unpublished data, 2003). ${ }^{6}$ An HPV type was described as "type $X$ " if a sample was positive by real time PCR yet negative by the LA assay.

\section{Prevalence measurements}

Women were stratified according to their concurrent cervical cytology grade, age, detection of LR-HPV or HR-HPV type(s), and presence of multiple HPV infections. The proportion of multiple HR-HPV infections was assessed in relation to the severity of cervical neoplasia and compared with data from patients harbouring a single HR-HPV type. HPV genotypes were ranked according to the frequency of detection in all positive samples, and then according to their frequency in cytologically negative, borderline dyskaryosis, mild dyskaryosis, and high grade dyskaryosis samples. Samples classified as having high grade dyskaryosis displayed either moderate or severe dyskaryosis, or evidence of suspected invasion.

\section{Statistical analysis of data}

When determining the prevalence of HR-HPV and LR-HPV types, women were counted more than once if they harboured a multiple infection with a mixture of both. The prevalence of individual HPV types was determined as they appeared as either single or within multiple infections. Multiple HPV infection was defined as two or more HPV types detected. Multiple HR-HPV infection was defined as two or more HR-HPV types, with or without additional LRHPV types. To examine the relation between HPV infection and the severity of cytological grade of cervical neoplasia, and to assess whether the difference in age between HPV positive and negative groups was significant, $\chi^{2}$ tests for linear trend and Mann-Whitney $U$ tests were performed. The strength of association of multiple HR-HPV infections, as opposed to single HR-HPV infection, with cytological grade of cervical neoplasia was analysed by a $\chi^{2}$ test. All statistics were performed using SPSS vll software.

\section{RESULTS}

\section{Cytological analysis of cervical samples}

Of the 3444 cervical samples analysed by HPV testing and cytological assessment of dyskaryosis, approximately $10 \%$ exhibited some degree of cytological neoplastic abnormality, including 3.3\% borderline dyskaryosis, 3.7\% mild dyskaryosis, and $2.7 \%$ high grade dyskaryosis. Only $0.5 \%$ of samples were graded unsatisfactory for cytological assessment (table 1).

\section{HPV prevalence}

HPV DNA was detected in 705 of 3444 samples tested (20\%). Of the HPV positive samples, 114 (16\%) were classified as type X. Thus, distribution trends of HR-HPV and LR-HPV types within the population were drawn from the remaining 591 that could be identified by the LA assay. Eligibility of the samples for HPV testing was confirmed by positive $\beta$ globin results.

As detailed in table 1, overall, HR-HPV types were more prevalent than LR-HPV types, even in individuals who had no cervical neoplasia detected. LR-HPV and HR-HPV types were detected in $187(26.5 \%)$ and $540(76.6 \%)$ of all 705 HPV positive individuals, respectively (representing 5.5\% and $15.7 \%$ of the overall population of 3444 , respectively). LRHPV types were detected in only 10 of 94 (10.6\%) of those with high grade dyskaryosis, always as part of a mixed infection with at least one other HR-HPV type. No exclusively LR-HPV infections were found in those with high grade dyskaryosis. The prevalence of unknown/untypable HPV

Table 1 Prevalence of HPV in Edinburgh and Lothian's routine cervical screening population

\begin{tabular}{|c|c|c|c|c|c|c|c|c|}
\hline & \multicolumn{8}{|c|}{ Prevalence of HPV } \\
\hline & $\begin{array}{l}\text { No. tested } \\
\text { (\% population ) }\end{array}$ & $\begin{array}{l}\text { HPV +ve } \\
(\%)\end{array}$ & $\begin{array}{l}\text { HPV -ve } \\
(\%)\end{array}$ & $\begin{array}{l}\text { HR-HPV +ve } \\
(\%)\end{array}$ & $\begin{array}{l}\text { HR-HPV +ve only } \\
\text { (\%) }\end{array}$ & $\begin{array}{l}\text { LR-HPV +ve } \\
(\%)\end{array}$ & $\begin{array}{l}\text { LR-HPV +ve } \\
\text { only (\%) }\end{array}$ & $\begin{array}{l}\text { Unknown HPV } \\
\text { type (\%) }\end{array}$ \\
\hline \multicolumn{9}{|l|}{ Dyskaryosis } \\
\hline Negative & 3089 (89.7) & 392 (12.7) & 2697 (87.3) & $257(8.3)$ & $200(6.5)$ & $93(3.0)$ & $36(1.2)$ & $99(3.2)$ \\
\hline Borderline & 114 (3.3) & 97 (85.1) & 17 (14.9) & 82 (71.9) & 53 (46.5) & 38 (33.3) & $9(7.9)$ & $6(5.3)$ \\
\hline Mild & $129(3.7)$ & $124(96.1)$ & $5(3.9)$ & $112(86.8)$ & $72(55.8)$ & $46(35.7)$ & $6(4.6)$ & $6(4.6)$ \\
\hline High grade & $94(2.7)$ & $88(93.6)$ & $6(6.4)$ & $86(91.5)$ & $76(80.8)$ & $10(10.6)$ & 0 & $2(2.1)$ \\
\hline $\mathrm{U} / \mathrm{S}$ & $18(0.5)$ & $4(22.2)$ & $14(77.8)$ & $3(16.6)$ & $3(16.6)$ & 0 & 0 & $1(5.5)$ \\
\hline \multicolumn{9}{|l|}{ Age } \\
\hline$<25$ & 734 (21.3) & $308(42.0)$ & $426(58.0)$ & $260(35.4)$ & $171(23.3)$ & $111(15.1)$ & $22(2.9)$ & $26(3.5)$ \\
\hline $25-35$ & $935(27.2)$ & $211(22.6)$ & 724 (77.4) & $158(17.0)$ & $127(13.6)$ & $44(4.7)$ & 13 (1.34) & $40(4.3)$ \\
\hline $35-45$ & $893(25.9)$ & $116(13.0)$ & $777(87.0)$ & $80(9.1)$ & $69(7.7)$ & $20(2.2)$ & $9(1.0)$ & $27(3.0)$ \\
\hline $45-55$ & 603 (17.5) & $55(9.1)$ & $548(90.9)$ & $33(5.5)$ & $29(4.8)$ & $8(1.3)$ & $4(0.7)$ & $18(3.0)$ \\
\hline$>55$ & $279(8.1)$ & $15(5.4)$ & 264 (94.6) & $9(3.2)$ & $8(2.9)$ & $4(1.4)$ & $3(1.1)$ & $3(1.1)$ \\
\hline Total & 3444 & 705 & 2739 & 540 & 404 & 187 & 51 & 114 \\
\hline
\end{tabular}

$\mathrm{HPV}$ +ve, all who tested HPV DNA positive (includes any individual who tested positive for HR-HPV and or LR-HPV type(s), in addition to those who were positive by PCR but untypable by the LA assay). HPV -ve, all who did not test HPV DNA positive. HR-HPV +ve, all who tested positive for at least 1 HR-HPV type (mixed infections with low risk types are included). HR-HPV +ve only, all who tested positive for a HR-HPV type or types only. LR-HPV + ve, all who tested positive for at least 1 LR-HPV type (mixed infections with high risk types are included). LR-HPV +ve only, all who tested positive for a LR-HPV type or types only. Unknown HPV type, all who tested positive for HPV using real time PCR but hybridised to none of the 27 probes in the LA assay.

$H P V$, human papillomavirus; HR, high risk; LA, linear array; LR, low risk; PCR, polymerase chain reaction; U/S, unsatisfactory. 
Table 2 Prevalence of multiple HPV infections according to cytological grade of dyskaryosis and age

\begin{tabular}{lccc}
\hline & $\begin{array}{c}\text { Multiple HPV } \\
\text { infection }\end{array}$ & $\begin{array}{c}\text { Total single } \\
\text { HR-HPV +ve }\end{array}$ & $\begin{array}{c}\text { Total multiple } \\
\text { HR-HPV +ve }\end{array}$ \\
\hline Dyskaryosis & & & \\
Negative & $139(4.5)$ & $152(5.0)$ & $105(3.4)$ \\
Borderline & $50(43.8)$ & $44(38.6)$ & $38(33.3)$ \\
Mild & $73(56.6)$ & $58(44.9)$ & $54(41.8)$ \\
High grade & $43(45.7)$ & $48(51.1)$ & $38(40.4)$ \\
U/S & $1(5.5)$ & 2 & $1(5.5)$ \\
Age & & & $147(20.0)$ \\
$<25$ & $186(25.3)$ & $113(15.4)$ & $61(6.5)$ \\
$25-35$ & $78(8.3)$ & $97(10.4)$ & $19(2.1)$ \\
$35-45$ & $28(3.1)$ & $61(6.8)$ & $7(1.2)$ \\
$45-55$ & $11(1.8)$ & $26(4.3)$ & $2(0.7)$ \\
$>55$ & $3(1.1)$ & $7(2.5)$ & 236 \\
Total & 306 & 304 & \\
\hline
\end{tabular}

Values are numbers of samples and percentages in parenthesis. Mutiple HPV infection, sample tested positive for $>1$ HPV type by LA assay. Total single HR-HPV + ve, sample tested positive for a single HR-HPV infection, either alone or with other LR-HPV types present. Total multiple HR-HPV + ve, samples tested positive for $>1$ HR-HPV type (sample could have contained LR-HPV types also).

HPV, human papillomavirus; HR, high risk; LA, linear array; LR, low risk; $\mathrm{U} / \mathrm{S}$, unsatisfactory.

infections when assessed separately from HR-HPV infections did not correlate with age or cytological status (table 1).

With increasing severity of cytological grade of dyskaryosis, there was an increasing prevalence of HR-HPV ( $p<0.001)$, with HR-HPV detected in 86 of 94 (91.5\%) of those with high grade dyskaryosis, compared with 112 of 129 (86.8\%) of mildly dyskaryotic and 82 of $114(71.9 \%)$ of borderline dyskaryotic samples (table 1). When LR-HPV and unknown HPV types were included in the HPV positive group, there was an association between the presence of HPV ("any" type) and evidence of dyskaryosis. Detection of HPV decreased with increasing age, with the highest prevalence (either HR-HPV, or LR-HPV, or "any" HPV) being found in women $<25$ years. The median ages of those who tested HPV positive (26.610 years) and negative ( 37.490 years) were significantly different $(p<0.001)$

\section{Multiple HPV infections}

The prevalence of multiple HPV infections in this population was high, with $43.3 \%$ of samples that were HPV positive hybridising to more than one HPV type on the LA assay (table 2; fig 1). The most common type of multiple HPV infection was HR-HPV types only (table 3), with 164 of 705 $(23.3 \%)$ (705 being the total number of HPV positive infections detected overall) falling within this category. HRHPV and LR-HPV types were found together in 136 of 705 (19.3\%), whereas exclusively LR-HPV multiple infections were only detected in six of $705(0.8 \%)$. Infections with

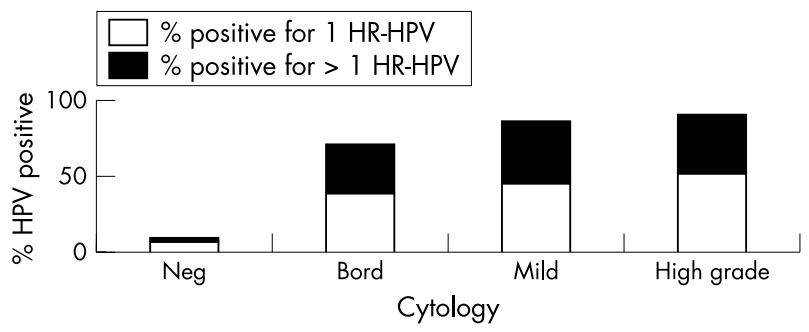

Figure 1 Prevalence of single and multiple high risk human papillomavirus (HR-HPV) infections associated with concurrent cytological grade of dyskaryosis. Neg, negative for dyskaryosis; Bord, borderline dyskaryosis; Mild, mild dyskaryosis; High grade, moderate or severe dyskaryosis or suspected invasion. multiple HR-HPV types were found in 105 of 3089 (3.4\%) samples negative for neoplasia, and in 38 of 114 (33.3\%), 54 of $129(41.8 \%)$, and 38 of $94(40.4 \%)$ samples with borderline, mild, or high grade dyskaryosis. There was no increased association (by $\chi^{2}$ test) between multiple HR-HPV infection and severity of neoplasia when compared with single HRHPV infections, which were identified in $152(4.9 \%)$ of negative samples, but in $44(38.6 \%), 58(45.0 \%)$, and 48 $(51.1 \%)$ patients with borderline, mild, or high grade dyskaryosis, respectively. Just under half of the HR-HPV infections were positive for more than one HR-HPV type across all cytological grades of dyskaryosis (fig 1). The prevalence of multiple HPV (all types) infections and multiple HR-HPV infections was also found to decrease with increasing age $(p<0.001)$ (table 2$)$.

\section{HPV type specific detection}

A broad diversity of infecting HR-HPV types was revealed with every HR-HPV type on the LA assay detected (table 4). Overall, the most abundant HR-HPV types were $16>18>51>31>52$, with HPV-16 detectable in 222 of $705(31.5 \%)$ HPV positive individuals, constituting a prevalence of $6.4 \%$ of the overall population. In contrast, HPV types 18, 51, 31, and 52 were detected in smaller proportions of samples, ranging from $2.2 \%$ to $1.8 \%$ of the overall population. When HR-HPV type specific infection was stratified by cytological grade of dyskaryosis, HPV-16 exhibited prevalences of 105 of $3089(3.4 \%), 69$ of 243 $(28.0 \%)$, and 46 of $94(48.9 \%)$ within samples with negative, low grade (borderline and mild dyskaryosis samples combined), and high grade dyskaryosis, respectively, whereas HPV-18 showed prevalences of $1.4 \%, 9.5 \%$, and $11.7 \%$ for these three groups. HPV-31 was the second most prevalent HR-HPV type in high grade dyskaryosis (17 of 94; 18.0\%). Interestingly, HPV-73 was found in $10.6 \%$ of high grade, but in only $5.8 \%$ of low grade dyskaryosis samples (table 3 ).

\section{DISCUSSION}

Because of the very low percentage $(0.5 \%)$ of cytologically unsatisfactory reports in this population, this work lends support to the use of LBC for cervical screening. Additional work on grading 17880 LBC samples collected from the same cervical screening population revealed only $0.6 \%$ as unsatisfactory, in contrast to $10 \%$ unsatisfactory samples when 8670 conventional Pap smears from the previous year were assessed (E McGoogan, unpublished data, 2003). These data imply that LBC screening can lead to reduced primary care

Table 3 Distribution of multiple infection containing HRHPV types exclusively, LR and HR-HPV types, and LR-HPV types exclusively

\begin{tabular}{lcll}
\hline & HR-HPV only & $\begin{array}{l}\text { HR-HPV and } \\
\text { LR-HPV }\end{array}$ & LR-HPV only \\
\hline Dyskaryosis & & & \\
Negative & $77(2.5)$ & $57(1.8)$ & $5(0.2)$ \\
Borderline & $20(17.5)$ & $29(25.4)$ & $1(0.9)$ \\
Mild & $33(25.6)$ & $40(31.0)$ & 0 \\
High grade & $33(35.1)$ & $10(10.6)$ & 0 \\
U/S & $1(5.5)$ & 0 & 0 \\
Age & & & \\
$<25$ & $93(12.7)$ & $89(12.1)$ & $4(0.5)$ \\
$25-35$ & $46(4.9)$ & $31(3.3)$ & $1(0.1)$ \\
$35-45$ & $17(1.9)$ & $11(1.2)$ & 0 \\
$45-55$ & $6(1.0)$ & $4(0.6)$ & $1(0.2)$ \\
$>55$ & $2(0.7)$ & $1(0.3)$ & 0 \\
Total & 164 & 136 & 6 \\
\end{tabular}

Values are numbers of samples and percentages in parenthesis. $\mathrm{HPV}$, human papillomavirus; HR, high risk; LR, low risk; U/S, unsatisfactory. 


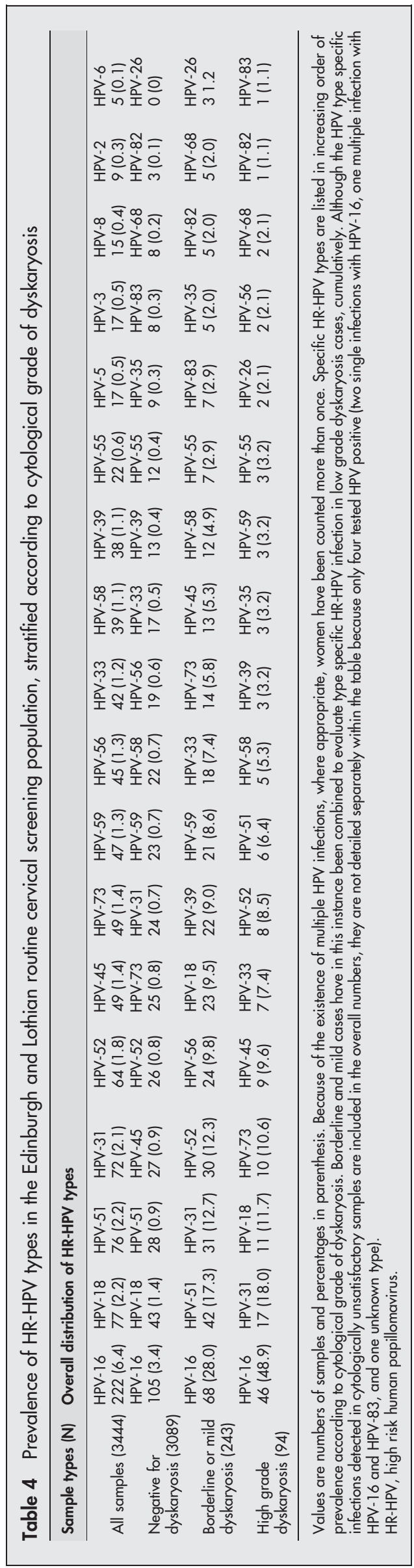

costs and a reduction in patient anxiety. ${ }^{7}$ The efficient turnaround time of LBC testing, coupled with application of the high throughput nucleic extraction system, as performed in our study, confirms the practicality of HPV testing in a routine setting.

From a global perspective, Edinburgh comprises a stable, largely white population with a low incidence of cervical cancer, estimated at 4.3 deaths/100 000. However, the overall prevalence of all HPV types in the population was quite high $(20 \%)$ and is close to that detected in "high risk" populations such as Colombia (14.9\%) and Paraguay $(20 \%) .{ }^{8}{ }^{9}$ However, estimates of HPV prevalence vary considerably, as shown in a USA study where $39.2 \%$ of 3863 women aged 18-40 attending a routine gynaecological clinic were HPV positive. ${ }^{10}$ The apparent disparity in prevalences strengthens the case for epidemiological evaluation on a population by population basis.

As with HR-HPV prevalence, LR-HPV prevalence was significantly higher in younger women, who usually have higher levels of sexual activity. ${ }^{11}{ }^{12}$ A second peak of HPV prevalence in peri-menopausal women was not evident in this population (as has been described previously, ${ }^{13}{ }^{14}$ ) and contrary to the findings of Chan et al, ${ }^{15}$ most HPV infections in this age group were not LR-HPV.

HR-HPV types were detected more frequently than LR-HPV types in women of all ages and grades of dyskaryosis. The diversity of HPV types was broad, and approximately $9.3 \%$ of the population tested positive for an HR-HPV type other than HPV-16 (found in 6.4\% of the population). Most of these infections were found in younger women and many are likely to be cleared. Yet, even in HPV positive women with high grade neoplasia, over $50 \%$ contained an HPV type or types other than HPV-16. More specifically, 29 of 1775 (1.6\%) women who were over 35 years within our study exhibited high grade neoplasia. Of these, 12 were infected with HPV16, 14 had an HR-HPV type or types other than HPV-16, and three were infected with type $X$, confirming a high prevalence and diversity of oncogenic HPV types.

\section{"We found that HPV-26, previously described as prob- ably carcinogenic, was the only type to be detected exclusively in cytologically abnormal samples, thereby supporting this classification"}

Unexpectedly, HPV-31 was found more commonly than HPV-18 in high grade dyskaryosis. HPV-73 was found in a similar proportion to HPV-18 in high grade neoplasia, consolidating its "high risk" status, as described in a recent article by Muñoz et al. ${ }^{16}$ In addition, we found that HPV-26, previously described as "probably carcinogenic", ${ }^{16}$ was the only type to be detected exclusively in cytologically abnormal samples, thereby supporting this classification. If HPV testing is to become incorporated into screening programmes, a broad spectrum test should be implemented until the true impact of the persistence of less common HR-HPV types in neoplastic progression is established.

The most striking feature that we noted was the high prevalence of multiple HR-HPV infections in all grades of cervical neoplasia. Currently, there is a lack of consensus within the literature about the extent and implications of multiple HPV infections, with reported prevalences varying from $17.5 \%$ in a gynaecological referral clinic population, ${ }^{10}$ to $46 \%$ in cancer biopsies, ${ }^{17}$ and $58.9 \%$ in women with low grade dysplasia. ${ }^{18}$ Human immunodeficiency virus (HIV) positive individuals are often infected with multiple HPVs, with reported figures of up to $80 \% .{ }^{19}$ Indeed, the detection of multiple HPV infections has been proposed by one group as a prognostic indicator for high grade neoplasia in HIV infected 


\section{Take home messages}

- There was a high prevalence of multiple high risk human papillomavirus (HR-HPV) infections in both high grade and low grade cervical neoplasia, reflecting common sexual transmission of multiple HR-HPV

- Multiple HR-HPV infections were most prevalent in young women, suggesting that greater sexual activity is associated with sexual transmission of multiple HR-HPV types

- Prospective cohort studies that link sequential loss or gain of HPV types with cytological analysis are required to assess the impact of multiple HR-HPV infections on neoplastic progression

women (U Weiland et al. Genital infections with multiple HPV types are frequent, are more associated with SIL and are more prevalent in HIV positive patients. Abstracts of the 20th International Papillomavirus Conference, Paris, France, 2002:75). In addition, in a study of immunocompetent women, multiple HPV infections were found to confer an increased odds ratio for risk of dysplasia that was second only to HPV-16 positivity. ${ }^{20}$ Significant differences in the mean number of HPV types detected between cytologically normal and dysplastic samples has also been reported..$^{21}$ In contrast, Rolón et al found no significantly higher risk of carcinoma in women with multiple HPV infections compared with those who were infected with a single HPV type.

"The key finding here of a high prevalence of multiple high risk human papillomavirus (HR-HPV) types in all grades of cervical neoplasia emphasises the lack of a cooperative carcinogenic relation between particular pairs or groups of HR-HPV types"

Cross sectional analysis of our data indicated that the detection of multiple HPV infections with HR-HPV types was not a significantly better predictor of high grade cervical neoplasia than single HR-HPV infection. Furthermore, multiple HR-HPV infections were more frequently found in younger women, who are more likely to be infected with HPV per se, suggesting that greater sexual activity is associated with sexual transmission of multiple HR-HPV types, perhaps more commonly than was previously thought. Thomas et al showed that no two HPV types were more or less likely to be acquired concurrently than any other two types. ${ }^{22}$ Rousseau et al suggested that persistence of HPV infection may be independent of coinfection with multiple HPV types at baseline, ${ }^{23}$ and Chaouki et al suggested that different HPV types did not act cooperatively in neoplastic transformation. ${ }^{24}$ Therefore, the key finding here of a high prevalence of multiple HR-HPV types in all grades of cervical neoplasia emphasises the lack of a cooperative carcinogenic relation between particular pairs or groups of HR-HPV types. This finding probably reflects the common sexual transmission of multiple HR-HPV types together. We hope the second phase of our longitudinal study will further address the relevance of multiple HR-HPV infections on the progression of cervical neoplasia.

\section{ACKNOWLEDGEMENTS}

The authors wish to thank Qiagen Ltd, UK and Roche Molecular Systems (specifically Dr J Kornegay), California for technical assistance with the nucleic acid extraction and molecular typing procedures, respectively. We are also grateful to P Campbell-Jones for her knowledge of PENS, and for helpful comments during the preparation of the manuscript, and to $\mathrm{C}$ Graham at the Welcome Trust Clinical Research Facility for support with the statistical analysis. The work was funded by the Chief Scientist Office of the Scottish Executive grant number CZB/4/33.

\section{Authors' affiliations}

K S Cuschieri, H A Cubie, A L Seagar, C Moore, E McGoogan, G Gilkisson, Royal Infirmary of Edinburgh, 51 Little France Crescent, Edinburgh EH16 4SA, UK

M W Whitley, St Triduanas Medical Practice, 54 Moira Park, Edinburgh EH7 6RU, UK

M J Arends, University of Cambridge Pathology Department, Addenbrooke's Hospital, Hills Road, Cambridge CB2 2QQ, UK

\section{REFERENCES}

1 Zielinski GD, Snijders PJ, Rozendaal L, et al. HPV presence precedes abnormal cytology in women developing cervical cancer and signals false negative smears. Br J Cancer 2001;85:298-404.

2 Kiaer SK, van den Brule, AC, Paull G, et al. Type specific persistence of highrisk human papillomavirus (HPV) as an indicator of high grade squamous intraepithelial lesions in young women: population based prospective follow up study. BMJ 2002;325:572-6.

3 Cuschieri KS, Seagar AL, Moore C, et al. Development of an automated extraction procedure for detection of human papillomavirus DNA in liquid based cytology samples. J Virol Methods 2003;107:107-13.

4 Cubie HA, Seagar AL, McGoogan EM, et al. Rapid real time PCR to distinguish between high risk human papillomavirus types 16 and 18. Mol Pathol 2001;54:24-9.

5 Gravitt PE, Peyton CL, Apple RJ, et al. Genotyping of 27 human papillomavirus types by using LI consensus PCR products by a singlehybridisation, reverse line blot detection. J Clin Microbiol 1998;36:3020-7.

6 Gravitt PE, Peyton CL, Alessi TQ, et al. Improved amplification of genital human papillomaviruses. J Clin Microbiol 2000;38:357-61

7 Whitley MW. Liquid based cytology: a success. BMJ 2003;327:161-2; author reply 162.

8 Molano M, Posso H, Weiderpass E, et al. Prevalence and determinants of HPV infection among Columbian women with normal cytology. $\mathrm{Br} J$ Cancer 2002;87:324-33.

9 Rolón PA, Smith JS, Muñoz N, et al. Human papillomavirus infection and invasive cervical cancer in Paraguay. Int J Cancer 2000;85:486-91.

10 Peyton CL, Gravitt PE, Hunt WC, et al. Determinants of genital human papillomavirus detection in a US population. J Infect Dis 2001;183:1554-64.

11 Burk RD, Kelly P, Feldman J, et al. Declining prevalence of cervicovaginal human papillomavirus infection with age is independent of other risk factors. Sex Transm Dis 1993;20:333-41.

12 Melkert PWJ, Hopman E, van den Brule AJC, et al. Prevalence of HPV in cytomorphologically normal cervical smears as determined by the polymerase chain reaction is age-dependent. Int J Cancer 1993;53:919-23.

13 Cuzick J, Berverley E, Ho L, ef al. HPV testing in primary screening of older women. Br J Cancer 1999;81:554-8.

14 Muñoz N, Kato I, Bosch FX, et al. Risk factor for HPV detection in middle-age women. Sex Transm Dis 1996;23:743-9.

15 Chan PKS, Chang AR, Cheung JLK, et al. Determinants of cervical human papillomavirus infection: differences between high and low-oncogenic risk types. J Infect Dis 2002;185:28-35.

16 Muñoz N, Bosch FX, de Sanjose S, et al. Epidemiologic classification of human papillomavirus types associated with cervical cancer. N Engl J Med 2003;348:518-27.

17 Bachtiary B, Obermair A, Dreier, et al. Impact of multiple infection on response to treatment and survival in patients receiving radical radiotherapy for cervical cancer. Int J Cancer 2002;102:237-43.

18 ALTS Group. Human papillomavirus testing for triage of women with cytologic evidence of low-grade squamous intraepithelial lesions: baseline data from a randomised trial. J Natl Cancer Inst 2000;92:397-402.

19 Levi JE, Kleter B, Quint WG, et al. High prevalence of human papillomavirus (HPV) infections and high frequency of multiple HPV genotypes in human immunodeficiency virus-infected women in Brazil. J Clin Microbiol 2002;9:3341-5.

20 Becker TM, Wheeler CM, McGrough NS, et al. Sexually transmitted diseases and other risk factors for cervical dysplasia among Southwestern Hispanic and non-Hispanic white women. JAMA 1994;271:1181-8.

21 Fife KH, Craner HM, Schroeder JM, et al. Detection of multiple human papillomavirus types in the lower genital tract correlates with cervical dysplasia. J Med Virol 2001;64:550-9.

22 Thomas KK, Hughes JP, Kuypers JM, et al. Concurrent and sequential acquisition of different genital human papillomavirus types. $J$ Infect Dis 2000; 182:1097-102.

23 Rousseau MC, Pereira JS, Prado JCM, et al. Cervical coinfection with human papillomavirus types as a predictor of acquisition and persistence of HPV infection. J Infect Dis 2001;184:1508-17.

24 Chaouki N, Bosch FX, Muñoz N, et al. The viral origin of cervical cancer in Rabat, Morocco. Int $J$ Cancer 1998;75:546-55. 